\title{
A New Technique for Reducing the Segmentation Error of Left Ventricle Contours using Magnetic Resonance Images
}

\author{
Amjad Khan \\ Dept. of Computing (HIET) \\ Hamdard University (HUIC) \\ Islamabad, Pakistan \\ amjadkhan_cs@yahoo.com \\ Lim Phei Chin \\ Dept. of Computing (FCSIT) \\ University Malaysia Sarawak (UNIMAS) \\ Sarawak, Malaysia \\ pclim@unimas.my
}

\author{
Dayang NurFatimah Awang Iskandar \\ Dept. of Computing (FCSIT) \\ University Malaysia Sarawak (UNIMAS) \\ Sarawak, Malaysia \\ dnfaiz@unimas.my
}

\author{
Wang Yin Chai \\ Dept. of Computing (FCSIT) \\ University Malaysia Sarawak (UNIMAS) \\ Sarawak, Malaysia \\ ycwang@unimas.my
}

\begin{abstract}
The number of Cardiac Magnetic Resonance Images produced for a patient is overwhelming, and this leads to several issues such as labour intensive, time-consuming and detected contours error. The current practice to evaluate the Cardiac Magnetic Resonance Images by the experts is either manual or semi-automatic. Ideally, an automatic evaluation is preferred to assist the cardiac experts in their clinical evaluation. The automatic segmentation for the left ventricle that is the endocardium (Endo) and epicardium (Epi) is currently lacking. The two most usable segmentation model approaches namely Level Set Model (LSM) and Variational LSM (VLSM) are very popular because of their fast iterating of the contours shape objects. Due to the unstructured LV shape and papillary muscles of the left ventricle, both models issue was reinitialisation on detected contours. In this paper presented, the combined Sign-Euclidean distance function takes the distance measurement, from centre to endocardium contour towards Epicontour. The distance measurement function using the distance mapping technique is guided by the curves line using energy function to reduce segmentation error. The experiments were conducted utilizing the Sunnybrook and Pusat Jantung Sarawak (PJS) cardiac datasets. The results shows that the Sign-Euclidean distance function reduces segmentation error between segmented contours, the highest error identified in Endo-contour is; HF-I05 (Endo-14.74); HF-NI-11 (Endo-8.79); P-A004 (Endo-8.04); in Epi-contours; HF-I-08 (Epi-3.08); HF-NI-07 (Epi-2.81); P-A001 (Epi-3.34). This paper aims to develop a combined Sign-Euclidean distance function that measures among segmented contours and reduces segmentation error against ground-truth contour.

Index Terms-segmentation error, distance measurement, distance mapping, energy function and cardiac contours.
\end{abstract}

\section{INTRODUCTION}

Segmentation approaches have been using as a key of developing for the prognosis and diagnosis of Cardiac Magnetic Resonance (CMR) disease including, Cardiac Magnetic Resonance-Left Ventricle (CMR-LV) contours detection, cardiac muscles extraction and area at risk (AAR) identification, healthy and pathological tissue classification and estimation of cardiac function. Physicians take a keen interest to figure out the cardiac contour size from the two cardiac cycle moments (Systolic and Diastolic). The Papillary Muscles and Trabeculation cause the prominent problem of the re-initialisation on detected contours using segmentation approach, which leads to missing of Left Ventricle (LV) contours. To solve the issue of re-initialisation od segmentation algorithm for counters is a very basic study was developed for CMR-LV Papillary Muscles removal include a band-stop filter method by a shift based clustering that partition the image into several harmonic regions technique and morphological filter operation, which detects the boundaries of papillary muscles removal [1]. Graph-cut geodesic anisotropic function was developed for inner contour segmentation [2]. Re-initialisation is a primary issue of segmentation error, highlighted [3]. Due to the issue of the weak edge detection, the model was modified by distance regularised level set function [4] and, the automatic function used to measure the distance between the Endo and Epi contour before the segmentation [5]. The initialization of VLSM selecting the Endo-contour objects by followed the distance measured function map, which forces the curves towards the initial contours and Epi-contour. ROI solution using a pattern recognition approach that had decomposed shape features of LV. This method adopts multi-dimensional machine learning techniques and designs new patterns for cardiac LV shapes. The patterns are classified into negative and positive shape features, the negative features were classified as Endo-contour features and positive features as Epi-contour. The automatic classifier identified these negative and positive shape features of CMR-LV based on a qualitative approach. The automatic classifier was adopted and used in developing Hough transform detector to automatically detects the shape features based on 